\title{
ON THE MODIFICATION OF DELANY AND BAZLEY FOMULAE
}

\author{
Ray Kirby \\ School of Engineering and Design, \\ Mechanical Engineering, \\ Brunel University, \\ Uxbridge, Middlesex, UB8 3PH, UK. \\ ray.kirby@brunel.ac.uk
}

The identification of the acoustic properties of porous materials is a very popular subject in the literature with numerous different approaches now available. One of the most popular is the methodology of Delany and Bazley [1], who studied a number of different fibrous materials and used regression formulae to quantify the bulk acoustic properties in the frequency domain. Delany and Bazley also showed that the bulk acoustic properties could be used to find the normal surface impedance of a sample of material, and hence its absorption coefficient. The method of Delany and Bazley is so effective because measurements are nondimensionalised and this permits extrapolation of the bulk acoustic properties outside of the parameters used during the original measurements. It is, however, well known that one should exercise caution when extrapolating Delany and Bazley’s formulae to low frequencies. Here, non physical data may be obtained and this may be seen, for example, in negative values for the real part of the surface impedance of a sample. Accordingly, at low frequencies some form of correction is required in order to provide physically consistent predictions for the properties of a porous material in the frequency domain. Many different 
approaches have been proposed for correcting Delany and Bazley’s formulae with varying degrees of complexity. One simple approach is the method proposed by Miki [2], which seems to be gaining some popularity, and also the related method by Komatsu [3]; it is the predictions provided by these two methods that will be discussed here.

Miki [2] and Komatsu [3] both propose heuristic modifications to Delany and Bazley’s formulae. Miki changes the regression coefficients but retains the structure of Delany and Bazley's equations, whereas Komatsu also makes changes to the fundamental structure of the equations, although the basic parameters remain the same. These corrections are designed to better reproduce Delany and Bazley’s original measured data at low frequencies, with the aim of avoiding non physical predictions for the surface impedance/absorption coefficient of a sample of fibrous material. The model of Miki has subsequently found some popularity, see for example Oliva and Hongisto [4], and at a recent conference attended by the author Miki’s method was mentioned on a number of occasions. In Miki’s original article the success of the proposed modifications was examined by comparing predictions against measured data obtained for the normal surface impedance of four different combinations of sample depth and material flow resistivity. In Komatsu's article the author chose to study the absorption coefficient, which is closely related to the surface impedance. Thus, both authors validate their new formulae only through the analysis of properties that depend on the depth of a finite sample of material, and in so doing neglect to investigate those fundamental properties of the material that are independent of material depth.

If one follows the traditional approach when applying Delany and Bazley’s formulae and assumes that a porous material may be treated as an equivalent fluid, then there are two fundamental properties for the material: the equivalent density and the speed of sound. 
Accordingly, any modifications to Delany and Bazley’s formulae that purport to avoid non physical behaviour at low frequencies must first be validated by examining the values they predict for the fundamental properties of the material. The equivalent fluid density $\rho$ and the sound speed $c$ may be written in terms of Delany and Bazley's coefficients in the following way

$$
\begin{gathered}
\hat{\rho}=-\left\{a_{1} \xi^{-a_{2}}+\mathrm{i}\left[1+a_{3} \xi^{-a_{4}}\right]\right\}\left\{a_{7} \xi^{-a_{8}}+\mathrm{i}\left[1+a_{5} \xi^{-a_{6}}\right]\right\} \\
\hat{c}=\left[1+a_{3} \xi^{-a_{4}}-\mathrm{i} a_{1} \xi^{-a_{2}}\right] /\left[1+2 a_{3} \xi^{-a_{4}}+a_{3}^{2} \xi^{-2 a_{4}}+a_{1}^{2} \xi^{-2 a_{2}}\right],
\end{gathered}
$$

where $\hat{\rho}=\rho / \rho_{0}, \hat{c}=c / c_{0}$ and $\xi=\rho_{0} f / \sigma$, with $f$ denoting frequency and $\sigma$ material flow resistivity. In addition, $i=\sqrt{-1}$, the subscript 0 denotes the ambient fluid properties, and Delany and Bazley's regression coefficients are $a_{1}=0.189, a_{2}=0.595 a_{3}=0.089, a_{4}=$ 0.7, $a_{5}=0.057, a_{6}=0.754, a_{7}=0.087, a_{8}=0.732$. The normal surface impedance $z$ for a sample of material of length $L$ may then expressed in terms of the fundamental parameters $\rho$ and $c$ in the following way

$$
z=\rho c \operatorname{coth}\left[\frac{\mathrm{i} 2 \pi f L}{c}\right]
$$

Accordingly, if one is to demonstrate the validity of modifications to Delany and Bazley's formulae it is necessary first to investigate how these modifications influence $\rho$ and $c$. Moreover, rather than investigate the real and imaginary parts of both $\rho$ and $c$, it is sufficient only to review the real part of the density, as it is here that any non physical behaviour can readily be seen. Therefore, in Fig. 1 the real part of the equivalent fluid density is shown, and this is calculated using Delany and Bazley’s original coefficients, as well as those of Miki [2] 
and Komatsu [3]. It is obvious in Fig. 1 why one should not extrapolate the data of Delany and Bazley below values of $\xi<0.01$, as this gives negative values for the real part of the equivalent fluid density. That is, if one extrapolates the data of Delany and Bazley to low values of $\xi$ (equivalent to low frequencies for a given sample of material) then non physical values for the equivalent density emerge. What is important in Fig. 1 is that Miki's model is also seen to deliver negative values for the real part of the equivalent density. In fact, Miki's model delivers non-physical predictions at even larger values of $\xi$ than that seen using Delany and Balzey's original formulae: the performance of Miki's formulae is actually worse than the formulae it is trying to correct. The method of Komatsu [3] does at least avoid negative values, but one can see that it delivers values that quickly become very large at relatively high values of $\xi$ and so it is highly unlikely that these are physically sensible estimations of the true behaviour of a porous material. Therefore, it can be concluded from Fig. 1 that the methods of Miki and Komatsu deliver non physical and/or implausible predictions for the equivalent density of a porous material.

The original aim of Miki [2] and Komatsu [3] was to avoid non physical predictions for the surface impedance and/or absorption coefficient of a sample of porous material. This may be achieved for certain combinations of material and sample depth $L$; however, because these methods were not validated for the fundamental properties of the material it is relatively straightforward to invent combinations of parameters in which problems arise with impedance predictions. For example, the normal surface impedance for a sample of depth $L=10 \mathrm{~cm}$ is shown in Fig. 2. Here Miki’s method is seen to provide non physical predictions for $\xi<0.001$, whilst Komatsu's method again avoids negative values but this is at the expense of rather large estimations of the real part of the impedance as $\xi$ is reduced. 
Fig. 2 illustrates why the model of Miki is attractive since it provides a superficially simple way of postponing the non physical predictions generated by Delany and Bazley’s formulae. But this is simply fortuitous, as there is no physical basis for the corrections provided by Miki, or those of Komatsu, and it is not difficult to show that their methods will not work for general combinations of material, frequency and sample depth.

The modifications proposed by Miki [2] and Komatsu [3] rely on modifying the structure and/or coefficients in Delany and Bazley's original formulae. There is no physical basis for doing this and the equations appear to have been developed only with simplicity in mind. The resulting equations are undeniably simple to apply and this probably explains the subsequent popularity of Miki’s approach. Fortunately, there are many alternative methods available in the literature that will avoid Miki’s non-physical predictions. For example, Mechel [5] retained a simple approach by specifying a new equation for use with values of $\xi<0.01$. A more physically complete approach demands an examination of the microstructure of the fibrous material, and Allard and Champoux [6] introduced a number of new parameters into a detailed microstructure model that delivers the correct limiting behaviour at low frequencies. Simplifications to this approach are also available, see for example Kirby [7] and Wilson [8], who both apply simplifications to a microstructure model and so avoid the need to measure those additional parameters introduced by Allard and Champoux. The methods of Kirby [7] and Wilson [8] are relatively straightforward and so they are compared against the predictions generated by Miki [2] and Komatsu [3] in Figs. 1 and 2. It is clear that both of these methods produce physically correct limiting behaviour at lower frequencies, and both methods require very little extra effort to implement when compared to those of Miki and Komatsu. Accordingly, it is concluded that authors seeking simple modifications suitable for correcting the low frequency behaviour of the Delany and 
Bazley formulae should not use the models of Miki [2] or Komatsu [3] and should instead seek out the many alternative methods available in the literature that deliver the correct limiting behaviour at low frequencies

\section{REFERENCES}

1. Delany ME, Bazley EN. Acoustical properties of fibrous materials. Applied Acoustics 1970;3:105-116.

2. Miki Y. Acoustical properties of porous materials - Modifications of Delany-Bazley models. Journal of the Acoustical Society of Japan 1990;11:19-24.

3. Komatsu T. Improvement of the Delany-Bazley and Miki models for fibrous soundabsorbing materials. Acoustical Science and Technology 2008;29:121-129.

4. Oliva D, Hongisto V. Sound absorption of porous material - Accuracy of prediction methods. Applied Acoustics 2013;74:1473-1479.

5. Mechel F.P. Ausweitung der absorberformel von Delany and Bazley zu tiefen frequenzen. Acustica 1976;35:210-213.

6. Allard J-F, Champoux. New empirical equations for sound propagation in rigid fibrous porous materials. Journal of the Acoustical Society of America 1992;91:3346-3353.

7. Kirby R, Cummings A. Prediction of the bulk acoustic properties of fibrous material at low frequencies. Applied Acoustics 1999;56:101-125.

8. Wilson D.K. Simple, relaxational models for the acoustical properties of porous media. Applied Acoustics 1997;50:171-188. 


\section{Figure Captions}

Figure 1. Real part of the normalised equivalent density. —— Delany and Bazley [1] ;

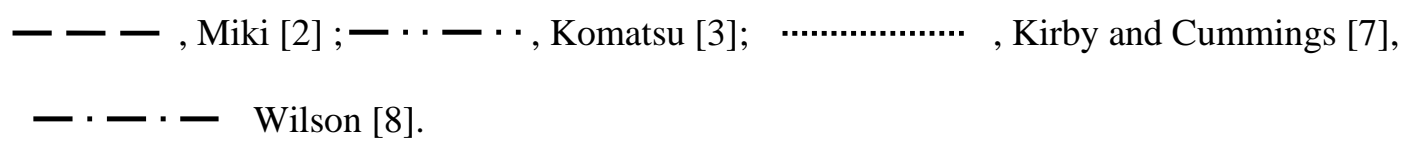

Figure 2. Real part of the normalised impedance. ——, Delany and Bazley [1] ;

— — , Miki [2] ; — . - . Komatsu [3];; ….............. , Kirby and Cummings [7],

- - - - Wilson [8]. 


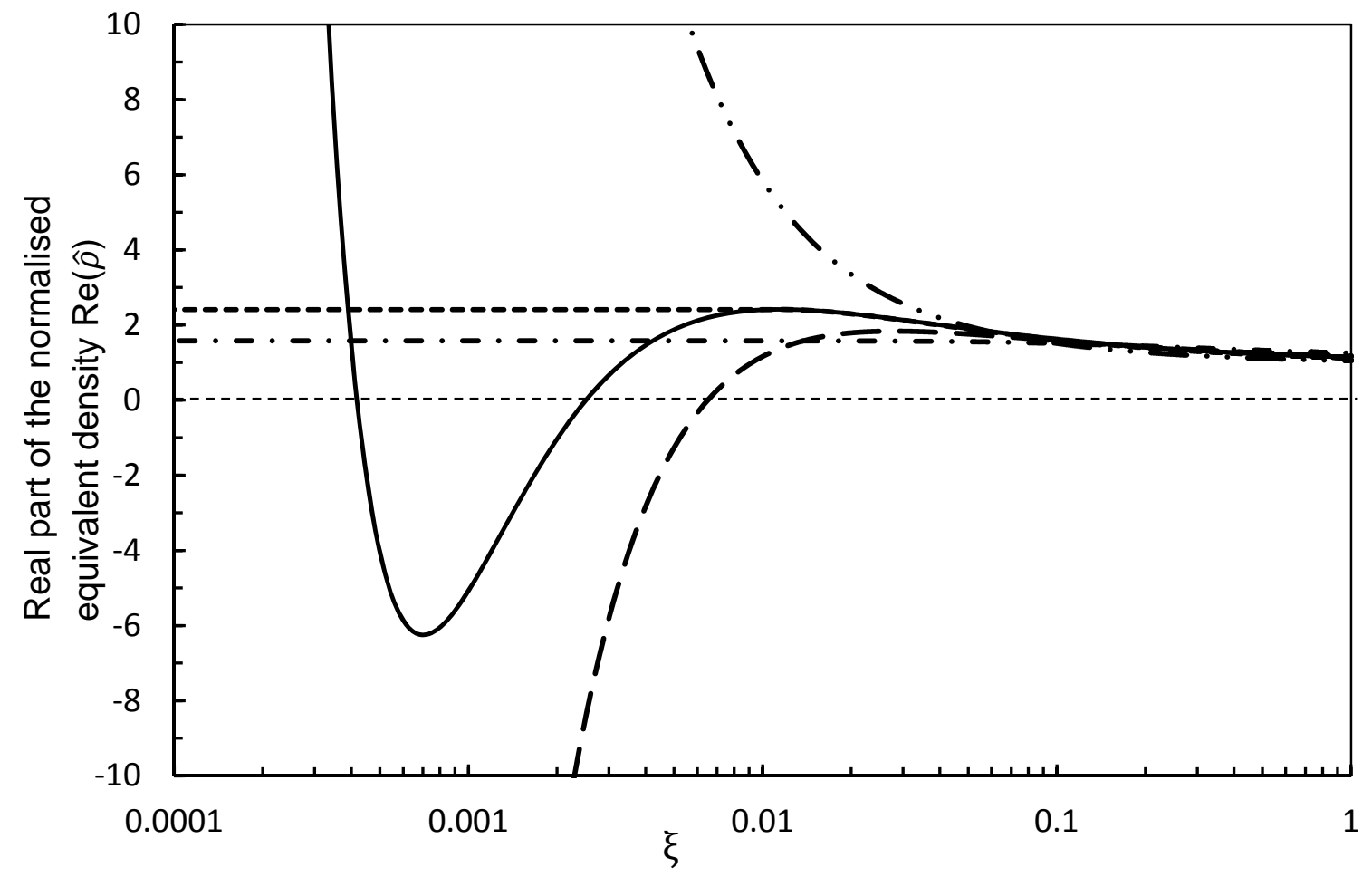

Figure 1. Real part of the normalised equivalent density. —— Delany and Bazley [1] ;

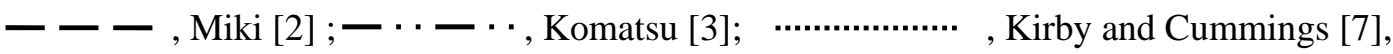
- - - - Wilson [8]. 


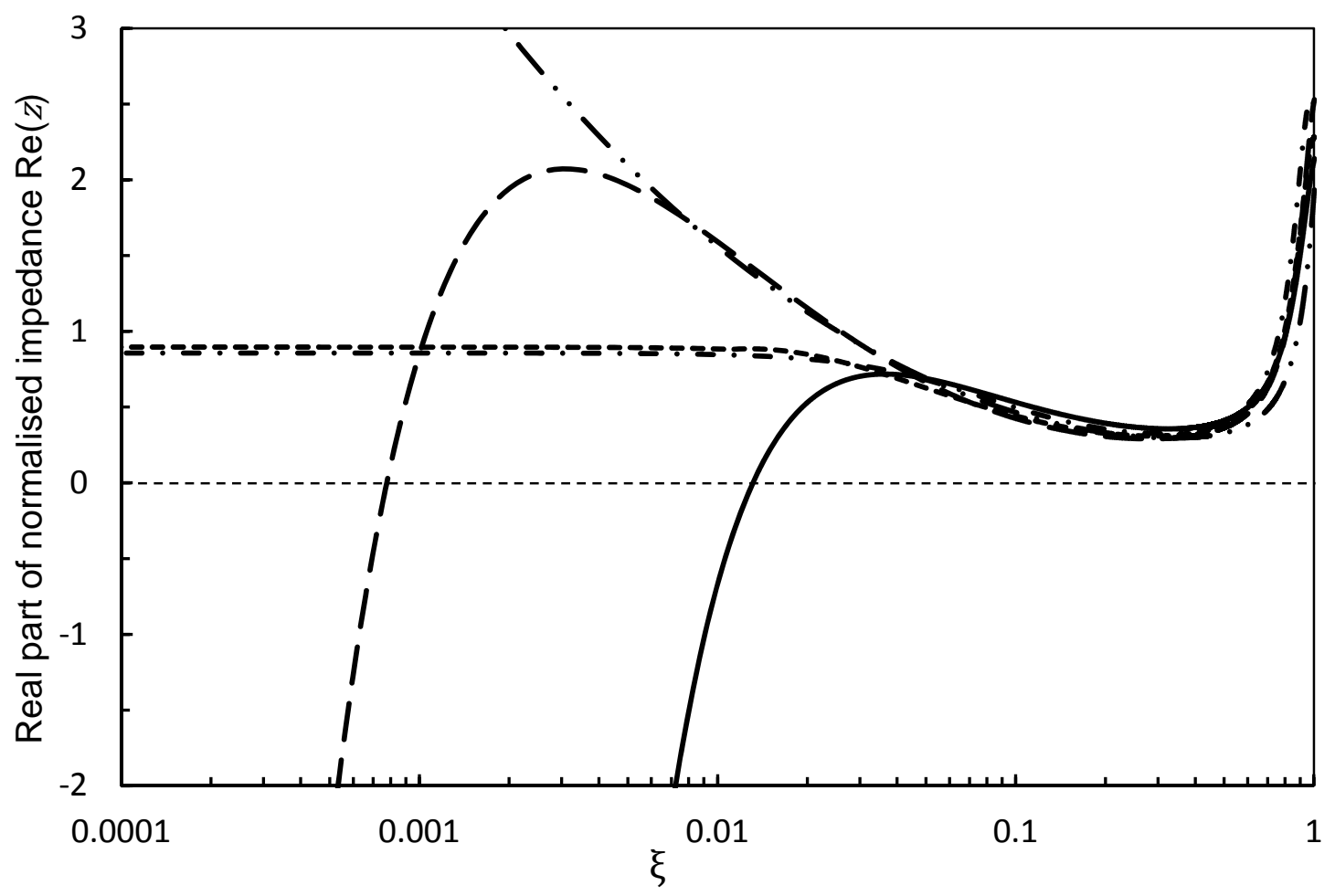

Figure 2. Real part of the normalised impedance. ——, Delany and Bazley [1] ;

- — - Miki [2] ; — . - . Komatsu [3];; ….............. , Kirby and Cummings [7], $-\cdot-\cdots$ Wilson [8]. 